\title{
Primordial fluctuations without scalar fields
}

\author{
João Magueijo and Johannes Noller \\ Theoretical Physics Group, Imperial College, London, SW7 $2 B Z$
}

(Dated: June 12, 2022)

\begin{abstract}
We revisit the question of whether fluctuations in hydrodynamical, adiabatical matter could explain the observed structures in our Universe. We consider matter with variable equation of state $w=p_{0} / \varepsilon_{0}$ and a concomitant (under the adiabatic assumption) density dependent speed of sound, $c_{s}$. We find a limited range of possibilities for a set up when modes start inside the Hubble radius, then leaving it and freezing out. For expanding Universes, power-law $w\left(\varepsilon_{0}\right)$ models are ruled out (except when $c_{s}^{2} \propto w \ll 1$, requiring post-stretching the seeded fluctuations); but sharper profiles in $c_{s}$ do solve the horizon problem. Among these, a phase transition in $c_{s}$ is notable for leading to scale-invariant fluctuations if the initial conditions are thermal. For contracting Universes all power-law $w\left(\varepsilon_{0}\right)$ solve the horizon problem, but only one leads to scale-invariance: $w \propto \varepsilon_{0}^{2}$ and $c_{s} \propto \varepsilon_{0}$. This model bypasses a number of problems with single scalar field cyclic models (for which $w$ is large but constant).
\end{abstract}

PACS numbers: 0000000

\section{INTRODUCTION}

Scalar fields are an easy tool for modeling the early universe, justifying their popularity. Unfortunately we have yet to detect a fundamental scalar field, and should one be found in high energy physics experiments this hardly proves their case in cosmology. In this paper we consider models for primordial structure formation based on hydrodynamical matter subject to certain thermodynamical constraints. Our construction is not dissimilar to that of 1, 2], where the concept of "generalized dark matter" - a parametrization of a generic hydrodynamical fluid - was introduced to explain the current cosmic acceleration. Our motivation, however, concerns the generation of a (quasi) scale-invariant spectrum of density fluctuations in the early universe.

Generating primordial density fluctuations entails a solution to the horizon problem, since the scales we now observe are initially causally disconnected according to the unreformed Big Bang model. A number of solutions have been proposed. Inflation is often invoked [3], and vacuum quantum fluctuations inevitably present in the modes inside the Hubble radius are transformed into near-scale invariant fluctuations near the deSitter phase. Other possibilities include the cyclic or ekpyrotic scenarios [4], string gas cosmology [5] and the varying speed of light (or sound) framework [6]. Combinations of these possibilities have also been considered (see for example [7]).

If we are to replace the proverbial scalar field with a hydrodynamical fluid the obvious question is what is its equation of state? In this paper we consider a power-law dependence between $w$ and density $\rho$. This is a feature of the popular Chaplygin gas and its modifications [8], but also of intermediate inflationary models [9, 10]. In fact the latter leads to a second solution for scale-invariance in the inflationary setting (the other solution being slowroll inflation [11]).

It is important to stress that hydrodynamical fluids and scalar fields are intrinsically different. Stress-energy fluctuations on scalar fields result from a cross term between zero and first order field fluctuations. The zeroth component is never thermalized even if the fluctuations are. In contrast, a thermal fluid behaves as a single unit, and so a number of thermodynamic theorems valid for fluids don't apply to scalar fields. This should be borne in mind throughout this paper (see [12] for an illustration of this point). The formalism of cosmological perturbations with a varying $w$ has been studied before but only in the context of radiation and matter mixtures (and multifluids more generally 18 20].)

The plan of our paper is as follows. In Section II we review results for the background solution with varying $w$ needed for the rest of this paper. Then in Section III we examine the hypothesis of adiabaticity and spell out its implications for the horizon and gauge problems. The formalism of cosmological perturbation theory for adiabatic fluctuations with a varying $w$ is developed in Section IV] A number of solutions are presented and discussed in Section V] and VI. A summary of our results is given in a concluding section.

Throughout this paper we shall assume that the fluid satisfies the adiabatic conditions. Non-adiabatic fluctuations are the subject of a paper in preparation [13].

\section{BACKGROUND SOLUTIONS WITH VARYING $w$}

To set the notation let the Friedmann equations be written as:

$$
\begin{gathered}
\mathcal{H}^{2}=\frac{1}{3} a^{2} \varepsilon_{0} \\
\mathcal{H}^{2}-\mathcal{H}^{\prime}=\frac{1}{2} a^{2}\left(\varepsilon_{0}+p_{0}\right) \equiv \mathcal{G},
\end{gathered}
$$

where $\mathcal{H}=a^{\prime} / a$ (with $a$ the expansion factor and a prime denoting derivative with respect to conformal time) and 
where we have defined the variable $\mathcal{G}$ in order to keep notation concise in what follows. The Friedmann equations imply a conservation equation for $\varepsilon$

$$
\varepsilon_{0}^{\prime}=-3 \mathcal{H}\left(\varepsilon_{0}+p_{0}\right) \text {. }
$$

These equations establish the background dynamics in units where $8 \pi G=1$. In order to solve them an equation of state $w=p_{0} / \varepsilon_{0}$ must be specified.

A number of background solutions with varying $w$ can be found in the literature. In this paper we shall focus on an adaptation of the equation of state [9]:

$$
1+w=\left(\frac{\varepsilon_{0}}{\rho_{\star}}\right)^{2 \beta},
$$

with $\beta>0$. This leads to exact solution:

$$
\begin{aligned}
a & =a_{0} \exp \left(t / t_{\star}\right)^{q}, \\
\varepsilon_{0} & =\frac{3 q^{2}}{t_{\star}^{2}}\left(\frac{t}{t_{\star}}\right)^{2 q-2}
\end{aligned}
$$

with parameters $q$ and $t_{\star}$ related to model parameters $\rho_{\star}$ and $\beta$ via:

$$
\begin{aligned}
q & =\frac{4 \beta}{1+4 \beta} \\
\rho_{\star} & =\frac{3 q^{2}}{t_{\star}^{2}}(2 \beta)^{\frac{1}{2 \beta}} \sim \frac{1}{t_{\star}^{2}} .
\end{aligned}
$$

The above solutions have been given in terms of proper time, rather than conformal time. The above excludes the case $\beta=0$, and $\beta=-1 / 4, q=-1 / 2$, that is $w \propto$ $1 / \sqrt{\varepsilon_{0}}$; but this case is outside our $\beta>0$ assumption anyway.

The regime $t \gg t_{\star}$ and $\varepsilon_{0} \ll \rho_{\star}$ corresponds to intermediate inflation, one of the two inflationary solutions for scale-invariant fluctuations 9 11. We are interested in $w \gg 1$ (associated with $c_{s} \gg 1$ in hydrodynamical fluids) and thus we'll focus on the opposite regime: $\varepsilon_{0} \gg \rho_{\star}$ and $t \ll t_{\star}$. More generally we can consider a variety of other models, with:

$$
w=w_{0}+\left(\frac{\varepsilon_{0}}{\rho_{\star}}\right)^{2 \beta}
$$

where $w_{0}$ is the fluid's low energy equation of state. At high density these models all have the same (power-law) behaviour. The high $w$ phase then exits into a constant $w$, low density phase which need not be inflation. We can also consider contracting models with these equations of state: an obvious generalization of cyclic models, where a high, but constant $w$ is invoked.

In the phase with $\varepsilon_{0} \gg \rho_{\star}$ and $w \gg 1$ we have that:

$$
\begin{aligned}
a & \approx a_{0} \\
\varepsilon_{0} & \propto t^{2 q-2} .
\end{aligned}
$$

This highlights the main peculiarity of these models. As a function of time the universe appears to be loitering $(a \approx$ $\left.a_{0}\right)$ and $a$ doesn't vanish at the Big Bang (although its time derivative diverges). However, its density changes like a power-law in $t$ and diverges as $t \rightarrow 0$. So although the scale factor doesn't vanish at $t=0$ we do have a Big Bang singularity. The fact that the metric and the matter change on different time-scales is behind a number of subtleties to be examined in this paper. Notice, for example, that conformal and proper time may be used interchangeably in many calculations.

\section{ADIABATIC FLUCTUATIONS AND THE HORIZON AND GAUGE PROBLEMS}

For the purpose of this paper a "solution of the horizon problem" means the existence of a phase in the early universe when modes are initially inside the Hubble radius (ruled by causal micro-physics), then cross outside to become dominated by gravity (i.e. "freezing-out"). This is the opposite kinematics to that realized in the standard Big Bang model. A number of solutions have been proposed, most notably inflation [3], ekpyrotic models [4], a Hagedorn phase [5] and a varying speed of light/sound [6]. Constant and varying $w$ models should be discussed separately if we examine the issue for adiabatic fluids.

Should $w$ and $c_{s}$ be constant, adiabaticity implies $w=c_{s}^{2}$, as is well known. This implies $w>0$, precluding inflation. Inflation can never be realized by a single adiabatic fluid, and it's not an accident that scalar fields are preferred. Thus, for adiabatic fluids, the horizon problem has to be solved with a contracting phase (e.g. in cyclic models [4]) or with a phase transition in the early Universe after a loitering epoch [5]. With a varying speed of sound, the adiabatic assumption requires a varying $w$ (in contrast with 6]) which we proceed to discuss.

If $w$ is variable, adiabaticity imposes a more complex relation than $w=c_{s}^{2}$. One can study the perturbation equations for modes inside the horizon (ignoring expansion) and derive the speed of propagation of pressure waves from first principles. This should be the rightful definition of the speed of sound $c_{s}$ and can be computed to be:

$$
c_{s}^{2}=\frac{\delta p}{\delta \varepsilon},
$$

where $\delta p$ and $\delta \varepsilon$ are the perturbed pressure and energy density. If this derivative is adiabatic, and if the background evolution is also adiabatic, then this implies the differential equation between $w$ and $c_{s}$ :

$$
c_{s}^{2}=\frac{d\left(w \varepsilon_{0}\right)}{d \varepsilon_{0}}=w+\varepsilon_{0} w_{, \varepsilon_{0}} .
$$

More generally (13) is true whenever the same equation of state $p=p(\varepsilon)$ is valid for fluctuations and unperturbed fluid (this isn't true, e.g. for scalar fields [12]). Note, however, that in non-adiabatic situations the correct definition for the speed of sound is (12) and not the definition 
given in terms of background quantities (e.g. [17]), which leads to (13).

Assuming that (13) is verified, if $w$ is a power law in $\varepsilon_{0}$ then we still have $c_{s}^{2} \propto w$ (even though $c_{s}^{2} \neq w$ ). The variation in $w$ can be ignored only if $c_{s}$ is a step function (or is ruled by a very large power). Thus the importance of investigating perturbations in varying $w$ fluids if one appeals to a varying $c_{s}$ to solve the horizon problem. More generally we can consider non-adiabatic phenomenological laws:

$$
\begin{aligned}
c_{s} & \propto \varepsilon_{0}^{\alpha} \\
w & \propto \varepsilon_{0}^{2 \beta}
\end{aligned}
$$

with the adiabatic case resulting from $\alpha=\beta$ and a suitable adjustment of the proportionality constants. Models with $\alpha \neq \beta$ will be the subject of a separate paper [13].

At this point we wish to highlight a subtlety, capable of explaining many of the results presented later in this paper. The gauge-problem in varying $w$ models becomes distinct from the horizon problem and imposes constraints on $\alpha$ and $\beta$. Usually sub-horizon modes do not suffer from the gauge-problem, whereas super-horizon modes do. Thus if the horizon problem has been solved, setting up initial conditions is gauge-invariant. By this we mean that it only involves scales for which gaugetransformations have negligible effect, so that different choices of gauge fixing (or different "gauge-invariant" options for $\delta \varepsilon$, for example), are equivalent (see [16] and specially [15]).

But in varying- $w$ models this is no longer true. Gauge transformations are generated by the Lie derivative of the background quantities [16, 18]. The background metric and matter variables now change on different time scales (cf. discussion at the end of Section III), specifically:

$$
\frac{\varepsilon_{0}^{\prime}}{\varepsilon_{0}} \sim w \frac{a^{\prime}}{a}
$$

and we highlight the extra $w$ factor in the right hand side. Therefore gauge transformations have negligible effect on different scales for metric and matter variables. For metric variables gauge transformations are irrelevant for modes satisfying:

$$
c_{s} k \gg \frac{a^{\prime}}{a}
$$

whereas for energy and pressure fluctuations the criterion is

$$
c_{s} k \gg \frac{a^{\prime}}{a} w
$$

If we don't want the initial conditions to be plagued by a gauge problem we now have a more stringent condition than the criterion for solving the horizon problem. For metric variables this reduces to $\alpha>1 / 2$, which is indeed the condition for solving the horizon problem. But for matter variables (in the models under consideration, described in Section (II) we have

$$
\alpha>\frac{1}{2}+2 \beta
$$

(we have used approximations, such as $a \approx$ const, peculiar to these models). This condition can never be met by adiabatic models.

The above assumes that the universe is expanding. If it is contracting the situation is reversed, as the universe gets denser and hotter in time rather than diluting and cooling. So it would appear that only contracting adiabatic models with varying $w$ can be used for structure formation, at least withing the models considered. This will indeed be our conclusion, after detailed calculations.

\section{THE LINEARIZED PERTURBATION EQUATIONS}

We now develop the machinery of cosmological perturbation theory with a varying $w$. Past literature has been restricted to matter-radiation and other fluid mixtures [18 20]. The generalization to any varying $w$ is straightforward but non-trivial, particularly if the adiabatic assumption is dropped.

The linearised perturbed Einstein equations for a perfect fluid stress-energy tensor of the form

$$
T_{\beta}^{\alpha}=(\varepsilon+p) u^{\alpha} u_{\beta}-p \delta_{\beta}^{\alpha}
$$

are

$$
\begin{gathered}
\Delta \Phi-3 \mathcal{H}\left(\Phi^{\prime}+\mathcal{H} \Phi\right)=\frac{1}{2} a^{2} \overline{\delta \varepsilon} \\
(a \Phi)_{, i}^{\prime}=\frac{1}{2} a^{2}\left(\varepsilon_{0}+p_{0}\right) \overline{\delta u}_{\|_{i}}=\mathcal{G} \overline{\delta u}_{\|_{i}}, \\
\Phi^{\prime \prime}+3 \mathcal{H} \Phi^{\prime}+\left(2 \mathcal{H}^{\prime}+\mathcal{H}^{2}\right) \Phi=\frac{1}{2} a^{2} \overline{\delta p} .
\end{gathered}
$$

where $\Phi$ is the Newtonian potential and the stress-energy fluctuations are evaluated in the longitudinal gauge (denoted by an overbar); we refer the reader to [20] for notation and further explanations. We use equation (22) to solve for $\Phi^{\prime}$ and $\Phi^{\prime \prime}$ :

$$
\Phi^{\prime}=\frac{\mathcal{G}}{a} \overline{\delta u}_{\|}-\mathcal{H} \Phi
$$

$$
\Phi^{\prime \prime}=\frac{\mathcal{G}}{a} \overline{\delta u}_{\|}^{\prime}+\frac{\mathcal{G}}{a}\left(\frac{p_{0}^{\prime}}{\varepsilon_{0}+p_{0}}-3 \mathcal{H}\right) \overline{\delta u}_{\|}+\mathcal{G} \Phi
$$

Combining equations (21) and (23), and considering that $c_{s}^{2}=\frac{\overline{\delta p}}{\overline{\delta \varepsilon}}$ we get the $\Phi$ equation:

$\Phi^{\prime \prime}+3\left(1+c_{s}{ }^{2}\right) \mathcal{H} \Phi^{\prime}+\left(2 \mathcal{H}^{\prime}+\left(1+3 c_{s}{ }^{2}\right) \mathcal{H}^{2}-c_{s}{ }^{2} \Delta\right) \Phi=0$. 
Using (24) and (25) to substitute in for $\Phi^{\prime}$ and $\Phi^{\prime \prime}$ we also derive the "equation of motion" for $\overline{\delta u}_{\|}$

$$
\overline{\delta u}_{\|}^{\prime}+\overline{\delta u}_{\|}\left(\frac{p_{0}^{\prime}}{\left(\varepsilon_{0}+p_{0}\right)}+3 c_{s}^{2} \mathcal{H}\right)=\left(1+\frac{c_{s}^{2} \Delta}{\mathcal{G}}\right)(a \Phi) \text {. }
$$

Equations (26) and (27) are the precursors for the equations for variables " $u$ " and " $v$ " favoured in the literature [19, 20]. For fluids $\Phi$ is related to " $u$ " and the velocity perturbation $\overline{\delta u}_{\|}$to " $v$ " (or the related curvature perturbation $\zeta$ ). These variables will be used in a future publication [13] to derive the second order action for the fluctuations with and without the adiabatic assumption. Here however we take another route.

Should we assume adiabatic fluctuations a number of simplifications are possible. Since for adiabatic fluctuations $c_{s}^{2}=\frac{p_{0}^{\prime}}{\varepsilon_{0}^{\prime}}$ we can derive the valuable identity:

$$
\frac{p_{0}^{\prime}}{\varepsilon_{0}+p_{0}}+3 c_{s}^{2} \mathcal{H}=\frac{w^{\prime}}{1+w}+3\left(c_{s}^{2}-w\right) \mathcal{H}=0 .
$$

Eqn. (26) can then be rewritten as an equation which for modes outside the horizon represents a conservation law. The "conserved quantity" is

$$
\zeta=\Phi \frac{5+3 w}{3(1+w)}+\frac{2}{3(1+w)} \frac{\Phi^{\prime}}{\mathcal{H}}
$$

and it's straightforward to prove that

$$
\zeta^{\prime}=-\frac{2}{3} \frac{c_{s}^{2} k^{2} \Phi}{\mathcal{H}(1+w)}
$$

is equivalent to (26) for any functional dependence of $w$. When the pressure is negligible we obtain a constant $\zeta$, as announced. The potential $\Phi$ can be eliminated altogether from this equation by taking another derivative. After a few algebraic manipulations, we get:

$$
\zeta^{\prime \prime}+2 \frac{z^{\prime}}{z} \zeta^{\prime}+c_{s}^{2} k^{2} \zeta=0
$$

with

$$
z \propto \frac{a(1+w)^{1 / 2}}{c_{s}}
$$

Defining variable $v$ by $\zeta=v / z$, this equation becomes the more familiar:

$$
v^{\prime \prime}+\left(c_{s}^{2} k^{2}-\frac{z^{\prime \prime}}{z}\right) v=0 .
$$

The quantity $v$ is the variable which is ruled by a scalar field action, when the second order action is evaluated.

Equations (31) and (33) reflect the tension between the dynamics for choices $\zeta$ and $v$ : For the first we have a friction term but no mass; for the latter no friction term and a (time-dependent) mass term. Sometimes it's easier to use one, sometimes the other. We shall keep both on the plate, and rather than examine a " $u$ " equation, infer all the information about the potential $\Phi$ from $\zeta$ and equations (29) or (30).

\section{EXPANDING SOLUTIONS}

We now consider solutions to these equations separating the cases of expanding and contracting Universes. In expanding Universes we find a no-go for all power-law models with large $w$ described in Section II. There are, however, other models which bypass this negative result, and display scale-invariant fluctuations. One example is a Universe with $c_{s}^{2}=w \ll 1$ (but this requires follow-up inflation or some other mechanism for stretching the size of the fluctuations). Another successful adiabatic model is a phase transition in $c_{s}$, provided the initial fluctuations are thermal.

\section{A. Absence of solutions for power-law $w$}

Were it not for the discussion on the gauge-dependence of pressure waves in Section III the following result might come as a surprise. We find that for the purpose of structure formation the horizon problem cannot be solved for all expanding adiabatic models with power-law $w\left(\varepsilon_{0}\right)$ and $w \gg 1$.

This can be easily seen from the analysis of the two competing terms in Eq. (33). If $w \gg 1$, then Eqn. (32) implies that $z \propto a$, so that the mass term is:

$$
\frac{z^{\prime \prime}}{z}=\frac{a^{\prime \prime}}{a}=\frac{1}{6} a^{2}\left(\varepsilon_{0}-3 p_{0}\right) \approx-\frac{1}{2} a^{2} w \varepsilon_{0} .
$$

But the adiabatic condition (13) applied to power-laws implies that $c_{s}^{2} \propto w$, as we've seen. Thus, for any pure power-law, the effect of a varying $c_{s}$ in the pressure term $c_{s}^{2} k^{2}$ is exactly canceled by the effect of $w$ on the varying mass term. Horizon crossing occurs at $k_{h}^{2} \sim a^{2} \varepsilon_{0}$ and the strong energy condition must be violated for this to increase in time. Specifically:

$$
\frac{\left(a^{2} \varepsilon_{0}\right)}{a^{2} \varepsilon_{0}}=-\frac{\dot{a}}{a}(1+3 w)>0
$$

so that $w<-1 / 3$ is needed.

\section{B. Near-dust with varying speed of sound}

It is possible to bypass this negative result in a number of cases, but the extra requirement of scale-invariance narrows down two possibilities.

One assumption that may be dropped is $w \gg 1$ (as was first pointed out in [21]; see also [7]). Specifically we could consider model (9) with $w_{0} \approx 0$ and $\beta>-1 / 2$ in the regime $\varepsilon_{0} \ll \rho_{\star}$. Then $z \propto a / c_{s}$ (i.e. $c_{s}$ and $w$ no longer cancel in $z$, as with $w \gg 1$ ). In addition $w$ is approximately constant $(w \approx 0)$ and so we fall into the regime already studied in [6]. It was shown that a solution for scale invariance is $c_{s} \propto \varepsilon_{0}$, so $\beta=1$ and $w=c_{s}^{2} / 5 \propto \varepsilon_{0}^{2}$. 
While this represents a solution to scale-invariant structure formation the scales produced are too small. This can be remedied by a mechanism stretching them following the varying $w$ phase that seeded the fluctuations. It was suggested in 21] that inflation might play such a role. But other methods of renormalizing the length scales could be attached to this model, such as a sharp drop in the speed of light [22], or a phase transition in the quantum structure of space-time [23].

\section{A phase transition in $c_{s}$}

If one drops the power-law assumption in (9) altogether, the argument against solving the horizon problem in expanding universes for adiabatic fluids breaks down. A number of solutions satisfying

$$
\left(\frac{c_{s}^{2} z}{z^{\prime \prime}}\right)^{\prime}<0
$$

are then possible. In general (13) does not imply $c_{s}^{2} \propto w$ : this is a peculiarity of power-laws. For example if $c_{s}$ undergoes a phase transition:

$$
\begin{aligned}
& \text { for } \varepsilon_{0}<\rho_{\star}: c_{s}=c_{s_{-}} \\
& \text {for } \varepsilon_{0}>\rho_{\star}: c_{s}=c_{s_{+}}
\end{aligned}
$$

with $c_{s_{+}} \gg c_{s_{-}}$, then the adiabatic condition (13) implies instead:

$$
\begin{aligned}
& \text { for } \varepsilon_{0}<\rho_{\star}: w=c_{s_{-}}^{2} \\
& \text { for } \varepsilon_{0}>\rho_{\star}: w=c_{s_{+}}^{2}+\left(c_{s_{-}}^{2}-c_{s_{+}}^{2}\right) \frac{\rho_{\star}}{\varepsilon_{0}} .
\end{aligned}
$$

Therefore $w$ can be assumed to be a constant while $c_{s}$ is sharply varying and Eqn. (32) implies $z \propto a / c_{s}$ (i.e. $c_{s}$ and $w$ don't cancel out in $z$ ). For $\varepsilon_{0} \gg \rho_{\star}$, the model satisfies (9) with $\beta=-1 / 2$; however, for $\varepsilon_{0} \approx \rho_{\star}$ (relevant when $c_{s}$ is varying and structures are being seeded) we can treat $w$ as a constant (as indeed $a$ and $\rho$ can be treated as constants). We then fall into the case already studied in [6]. The horizon problem is solved: modes start inside the horizon. If their initial conditions are set by the zero-point vacuum fluctuations we obtain a blue spectrum, with $n_{s}=2$; but if thermal initial conditions are used, we get scale invariance [6].

There are other non-power law solutions for $w$ and $c_{s}$ that solve the horizon problem for adiabatic fluids: for them the effect of $c_{s}$ in the pressure term no longer cancels with that of $w$ in $z^{\prime \prime} / z$. In general Eqn. (13) has a homogeneous solution, i.e. a $w$ profile which has no effect on $c_{s}$. This is a Chaplygin-type of gas, with $w=A / \varepsilon_{0}$. It is precisely this solution that appears linking the two constant $c_{s}$ phases in the phase transition scenario.

\section{A SOLUTION FOR CYCLIC SCENARIOS}

The situation described in the previous section is reversed should the universe be contracting, such as in cyclic models. Then for all power-law models described in Section II modes start inside the horizon, then leaving and freezing out. It's not difficult to work out the condition for approximate scale-invariance.

\section{A. The set up for the calculation}

A calculation similar to that in [6] can be applied to these models. However, the usual Bessel solutions, interpolating between inside and outside the horizon regimes, are no longer valid. Fortunately matching these regimes is enough for obtaining the spectrum and amplitude of fluctuations left outside the horizon.

While the pressure term dominates the solution has the WKB form:

$$
v \sim \frac{e^{i k \int c_{s} d \eta}}{\sqrt{2 c_{s} k}},
$$

which acts as a boundary condition. The normalization ensures that, upon second quantization, amplitudes multiplying this solution become creation and annihilation operators. As the Universe contracts and $\varepsilon_{0}$ increases, the modes become dominated by the term in $z^{\prime \prime} / z$ in equation (33) (c.f. the argument given in Section VA). In this regime the general solution for (31) (or for (33)) is:

$$
\zeta=\frac{v}{a}=A+B \int \frac{d \eta}{a^{2}} \approx A+\frac{B}{a_{0}^{2}}|\eta|,
$$

where $A$ and $B$ are (possibly $k$ dependent) constants, and we have used the discussion in Section II (cf. Eqn. (5)) in the last approximation (note also that $z \propto a$ approximately).

Usually the growing and decaying solutions are reversed in a contracting universe, and the constant, frozen-in mode becomes sub-dominant, while the time dependent mode diverges. This doesn't happen here: the term in $B$ actually goes to zero as we approach the crunch. Throughout $\zeta \sim v$ since $a$ changes by at most by a factor of 2 throughout the relevant phase.

\section{B. Conditions for (near) scale-invariance}

The power spectrum left outside the horizon (fixed by factor $A$ ) can be found by gluing (41) and (42) at horizon crossing. Horizon crossing occurs when the two terms in $v$ in (33) are of the same order, i.e. when $c_{s}^{2} k^{2}=\left|z^{\prime \prime} / z\right|$. Using approximations valid when $w \gg 1$ we have $k_{h}^{2} \propto$ $a^{2} w \varepsilon_{0} / c_{s}^{2}$ so that $k_{h} \propto \sqrt{\varepsilon_{0}}$ for the gluing point. We take the ansatz

$$
\zeta(k, \eta) \approx v(k, \eta) \propto k^{\frac{n_{s}}{2}-2}
$$

for the constant $v$ left outside the horizon (in general this need not be a power-law in $k$ ). This should be glued to:

$$
v \sim \frac{1}{\sqrt{c_{s} k}}
$$


when $k=k_{h} \propto \sqrt{\varepsilon}_{0}$. Writing the resulting identity in terms of $\varepsilon_{0}$ therefore produces the relation $\alpha+n_{s} / 2-$ $3 / 2=0$. Gluing therefore implies the expression for the spectral index:

$$
n_{s}=3-2 \alpha
$$

(up to corrections logarithmic in $k$ ). The condition for scale-invariance is $\alpha=1$, that is $c_{s} \propto \varepsilon_{0}$ (just like in models with constant $w$ discussed in 6]). The model associated with scale-invariance is therefore characterized by the high density equation of state:

$$
c_{s}^{2}=3 w=3\left(\frac{\varepsilon_{0}}{\rho_{\star}}\right)^{2} .
$$

It has expansion factor and density profile of the form:

$$
\begin{aligned}
a & =a_{0} \exp \left(|t| / t_{\star}\right)^{4 / 5}, \\
\varepsilon_{0} & =\frac{3(4 / 5)^{2}}{t_{\star}^{2}}\left(\frac{t_{\star}}{|t|}\right)^{2 / 5} .
\end{aligned}
$$

The amplitude of the fluctuations is tuned by parameter $\rho_{\star} / M_{p l}^{4}$.

The above assumes a vacuum expectation value, producing a constant, $k$-independent factor when creation and annihilation operators are inserted in second quantized solutions. If instead a thermal state is taken as a boundary condition, then a factor of $T_{c} / k$ multiplies the spectrum, with $T_{c}=T a$ the conformal temperature (cf. [6, 12]). Reworking the result therefore leads to a spectrum with index $n_{s}=2(1-\beta)$. Scale invariance under thermal fluctuations thus requires $\beta=1 / 2$ (which is the model leading to intermediate inflation, but in a totally different regime). We have $c_{s} \propto \sqrt{\varepsilon_{0}}$ and $c_{s}^{2}=w$ and the model is characterized by:

$$
\begin{aligned}
a & =a_{0} \exp \left(t / t_{\star}\right)^{3 / 2}, \\
\varepsilon_{0} & =\frac{3(4 / 5)^{2}}{t_{\star}^{2}}\left(\frac{t_{\star}}{t}\right)^{2 / 3} .
\end{aligned}
$$

Again the amplitude of the fluctuations is tuned by $\rho_{\star} / M_{p l}^{4}$, as well as $T_{c}$.

\section{Advantages over more standard cyclic scenarios}

The above has obvious similarities with traditional cyclic models 25]. In these $w \gg 1$ and $c_{s}=1$; here both $w$ and $c_{s}$ are large and vary like power-laws of the energy density. Cyclic models, in their original formulation, have a number of possible problems [25]. It's the potential $\Phi$ that's scale-invariant, not $\zeta$, which has a blue spectrum, with $n_{s}=3$. In addition $\Phi$ is not constant, but diverges like $1 /|\eta|$. Recent models, with multiple scalar fields have attempted to fix these shortcomings [26].

In desirable contrast, in the model we have proposed $\zeta$ is constant and scale-invariant. However the spectrum of $\Phi$ is not scale-invariant. In order to obtain $\Phi$ we use (30) for which we need to know the next order expansion in $\zeta$. This is:

$$
\zeta=A k^{-3 / 2}\left(1+C_{1}(k \eta)^{2}+C_{2} k \eta\right) .
$$

The first term comes from the first order corrections induced by a WKB inclusion of the pressure terms; the second is the decaying mode calculated for our model in (42). For any model the Taylor expansion in $k \eta$ will have two such leading terms. If the first term dominates, $\zeta$ and $\Phi$ have the same spectrum (as in inflation and the varying speed of sound scenarios in [6, 24] ). When the second term dominates $\Phi$ and $\zeta$ become quite different, such as in cyclic scenarios, and here. Inserting (51) in (30) implies that

$$
\Phi \propto k^{-5 / 2} \mathcal{H}
$$

so that the spectral index for $\Phi$ is $n_{s}=-1$ (a red spectrum) and it diverges like $\mathcal{H} \sim \sqrt{\varepsilon_{0}}$ as we approach the crunch.

The situation is similar, but slightly better, to that of a collapsing matter Universe [27]. In that case fluctuations in $\zeta$ are scale-invariant, but those in $\Phi$ are red (with $n_{s}=-3$ ). However in such models severe fine tuning afflicts the amplitude, because the fluctuations in $\zeta$ and $\Phi$ diverge as we approach the crunch. That problem doesn't plague out model, at least if we adopt the view that it's the fluctuations in $\zeta$ that matter. Still, the potential behaves somewhat pathologically.

Needless to say that this model shares with all other cyclic scenarios the usual uncertainties about the transmission of the spectrum past the bounce.

\section{CONCLUSIONS}

We considered fluctuations in adiabatic hydrodynamical models with varying $w$. Our results can be summarised as follows.

Under the adiabatic assumption, inflation can never be realized (since it implies $w>0$ for any sustained period). But neither can the simplest realizations of the varying speed of sound mechanism: power-law equations of state in expanding universes. For adiabatic fluids $c_{s}^{2}$ and $w$ must then be proportional. The variation in $w$ has effects on the varying mass term $z^{\prime \prime} / z$ describing the effects of expansion. The effect of $c_{s}$ on the pressure cancels with the effect of $w$ on $z^{\prime \prime} / z$ and so the horizon problem is in fact never solved.

To bypass this result in expanding Universes one must drop the assumption that $w$ is a power-law. A sharp phase transition in $c_{s}$ leads to scale-invariance if the initial conditions are set by a thermal state. A model with $w \ll 1$ and vacuum fluctuations is also a possibility, but the scales produced must then be stretched by an external mechanism. 
For contracting universes the vistas expand. Specifically, in cyclic and ekpyrotic models, we find that the law $c_{s}^{2}=3 w \propto \rho^{2}$ leads to scale invariant fluctuations. Some of the problems in the ekpyrotic scenario are even bypassed. Scale-invariance is achieved in variable $\zeta$ and is present in both of its modes. The amplitude of the fluctuations in $\zeta$ freezes-in, instead of diverging. However the spectrum of fluctuations in $\Phi$ is red and its amplitude time-dependent. The problem of the transmission of these spectra to life after the bounce remains open and unsolved.

One may be rightly concerned that causality paradoxes afflict these models, given that $c_{s}>1$ is permitted, a matter that affects any varying speed of sound scenario. As discussed in 24] a resolution of these problems is achieved by a bimetric reformulation. The larger speed of sound in the gravity frame then simply signals the presence of a non-conformal matter metric. In previous work (based on the anti-DBI action 24]) the re-examination of the model in terms of two metrics only reinforced its motivation, as it resulted from the minimal bimetric theory. Examining the bimetric structure behind the models discussed here is therefore relevant, but beyond the scope of this paper. In a future publication we shall examine the status of perfect fluids in bimetric theories where the bi-scalar is a spectator field [14]. In another forthcoming paper [13] we shall also examine the implications of dropping the assumption of adiabaticity pervading this paper.

Acknowledgments We'd like to thank an anonymous referee for very helpful comments and for correcting several typos and STFC for financial support.
[1] W. Hu, Astrophys.J. 506: 485, 1998.

[2] J. Weller and A. Lewis, M.N.R.A.S. 346: 987, 2003.

[3] A. Guth, Phys.Rev. D23 347 (1981); A. Linde, Phys. Lett B 108, 1220 (1982).

[4] P. Steinhardt and N. Turok, Science 296: 1436-1439, 2002.

[5] A. Nayeri, R. Brandenberger and C. Vafa, Phys. Rev. Lett.97: 021302, 2006.

[6] J. Magueijo, Phys. Rev. Lett. 100, 231302 (2008).

[7] J. Khoury and F. Piazza, arXiv:0811.3633.

[8] N. Billic, G. Tupper and R. Viollier, Phys.Lett. B535, 17,2002 ; M. Bento, O. Bertolami and A. Sen, Phys.Rev. D66: 043507, 2002.

[9] J. Barrow, Phys. Lett B235, 1990.

[10] J. Barrow and A. Liddle, Phys.Rev. D47, 1993; Phys.Rev.D74:127305,2006.

[11] A. Starobinsky, JETP Lett. 82,2005.

[12] P. Ferreira and J. Magueijo, Phys.Rev. D78: $061301,2008$.

[13] J. Noller and J. Magueijo, 0911.1907.

[14] J. Noller and J. Magueijo, in preparation.

[15] R. Durrer, Phys. Rev. D 42, 2533 (1990).

[16] J. M. Stewart, Class.Quant.Grav. 7: 1169-1180, 1990.

[17] J. Bardeen, Phys. Rev. D 22, 1882 (1980).
[18] H. Kodama and M. Sasaki, Prog. Theor. Phys. Suppl.78: 1-166, 1984.

[19] J. Lidsey et al, Rev. Mod. Phys. 69: 373-410, 1997.

[20] S. Mukhanov, H. Feldman and R. Brandenberger, Phys.Rept. 215: 203-333, 1992; Physical Foundations of Cosmology, V. Mukhanov, CUP (2005).

[21] C. Armendariz-Picon and E. Lim, JCAP 0312: 002, 2003; C. Armendariz-Picon JCAP 0610: 010, 2006.

[22] A. Albrecht and J. Magueijo, Phys.Rev. D 59043516 (1999).

[23] J. Magueijo, L. Smolin and C. Contaldi, Class.Quant.Grav.24: 3691-3700, 2007.

[24] J. Magueijo, Phys.Rev.D79: 043525, 2009.

[25] S. Gratton et al, Phys.Rev. D69 (2004) 103505.

[26] E. I. Buchbinder, J. Khoury and B. A. Ovrut, Phys. Rev. D 76, 123503 (2007); J. Lehners et al. Phys. Rev. D 76, 103501 (2007); P. Creminelli and L. Senatore, JCAP 0711, 010 (2007); K. Koyama and D. Wands, JCAP 0704, 008 (2007); K. Koyama, S. Mizuno and D. Wands, Class. Quant. Grav. 24, 3919 (2007).

[27] F. Finelli and R. Brandenberger, Phys. Rev. D 65, 103522 (2002); D. Wands, Phys. Rev. D 60, 023507 (1999). 